\title{
Monte Carlo Methods in Climate Science
}

\author{
John C. Baez \\ Department of Mathematics, University of California \\ Riverside, CA 92521 USA \\ David Tweed \\ Azimuth
}

September 19, 2013

\section{Introduction}

They look placid lapping against the beach on a calm day, but the oceans are actually quite dynamic. The ocean currents act as 'conveyor belts', transporting heat both vertically between the water's surface and the depths and laterally from one area of the globe to another. This effect is so significant that the temperature and precipitation patterns can change dramatically when currents do.

For example: shortly after the last ice age, northern Europe experienced a shocking change in climate from 10,800 to $9,500 \mathrm{BC}$. At the start of this period temperatures plummeted in a matter of decades. It became $7^{\circ}$ Celsius colder, and glaciers started forming in England! The cold spell lasted for over a thousand years, but it ended as suddenly as it had begun.

Why? The most popular theory is that a huge lake in North America formed by melting glaciers burst its bank - and in a massive torrent lasting for years, the water from this lake rushed out to the northern Atlantic ocean. By floating atop the denser salt water, this fresh water blocked a major current: the Atlantic Meridional Overturning Circulation. This current brings warm water north and helps keep northern Europe warm. So, when it shut down, northern Europe was plunged into a deep freeze.

Right now global warming is causing ice sheets in Greenland to melt and release fresh water into the North Atlantic. Could this shut down the At- lantic Meridional Overturning Circulation and make the climate of Northern Europe much colder? In 2010, Keller and Urban [4] tackled this question using a simple climate model, historical data, probability theory, and lots of computing power. Their goal was to understand the spectrum of possible futures compatible with what we know today.

Let us look at some of the ideas underlying their work.

\section{Box models}

The earth's climate is too complex to simulate from the bottom up using basic physical principles, at least for now. The most detailed models we have use simplifying assumptions - and even so, they take days to run on very powerful computers. So, to make reasonable predictions on a laptop in a tractable time-frame, geophysical modellers need to use some clever tricks.

First, it is possible to split geophysical phenomena into 'boxes' containing only strongly related things. For example: atmospheric gases, particulate levels and clouds all affect each other strongly; likewise the heat content, currents and salinity of the oceans all interact strongly. However, the interactions between the atmosphere and the oceans are weaker, and we can approximately describe them using just a few parameters, such as the amount of atmospheric $\mathrm{CO}_{2}$ entering or leaving the oceans. Clearly these interac- 
tions must be consistent-for example, the amount of $\mathrm{CO}_{2}$ leaving the atmosphere box must equal the amount entering the ocean box-but breaking a complicated system into parts lets different specialists focus on different aspects; then we can combine these parts and get an approximate model of entire planet. The box model used by Keller and Urban is shown in Figure 1.

Second, it turns out that simple but effective box models can be distilled from the complicated physics in terms of forcings and feedbacks. A forcing is a measured input to the system, such as solar radiation or $\mathrm{CO}_{2}$ released by burning fossil fuels. As an analogy, consider a child on a swing: the adult's push every so often is a forcing. A feedback describes how the current state of our system influences its future state. In the swing analogy, one feedback is how the velocity will influence the child's future height. Specifying feedbacks typically uses knowledge of the detailed low-level physics to derive simple, tractable functional relationships between groups of large-scale observables, a bit like how we derive the physics of a gas by thinking about collisions of lots of particles.

However, it is often not feasible to get actual settings for the parameters in our model starting from first principles. In other words, often we can get the general form of the equations in our model, but they contain a lot of constants that we can estimate only by looking at historical data.

\section{Probability modeling}

Suppose we have a box model that depends on some parameters $S$. For example, in Keller and Urban's model, $S$ is a list of 18 numbers. To keep things simple, suppose $S$ is an element of some finite set. Suppose we also have huge hard disc full of historical measurements, and we want to use this to find the best estimate of $S$. Because our data is full of 'noise' from other unmodeled phenomena, we usually cannot unambiguously deduce a the correct value of $S$. Instead we have to look at things in terms of probabilities. More precisely, we need to study the probability that $S$ take some value $s$ given that the measurements take some value. Let's call the sequence of measurements $M$, and again let's keep things simple by saying that $M$ takes values in some finite set of possible measurements.

The probability that $S=s$ given that $M$ takes some value $m$ is called the conditional probability $P(S=s \mid M=m)$. How can we compute this conditional probability? This is a somewhat tricky problem.

One thing we can more easily do is repeatedly run our model with randomly chosen settings and see what measurements it predicts. By doing this, we can compute the probability that given setting values $S=s$, the model predicts measurements $M=m$. This again is a conditional probability, but now it is called $P(M=m \mid S=s)$.

This is not what we want: it's backwards! But here Bayes' rule comes to the rescue, relating what we want to what we can more easily compute:

$$
P(S=s \mid M=m)=P(M=m \mid S=s) \frac{P(S=s)}{P(M=m)}
$$

Here $P(S=s)$ is the probability that the parameters take a specific value $s$, and similarly for $P(M=m)$. Bayes' rule is quite easy to prove, and it is actually a general rule that applies to any random variables, not just the parameters and the measurements in our problem [7]. It underpins most methods of figuring out hidden quantities from observed ones. For this reason, it is widely used in modern statistics and data analysis [5].

How does Bayes' rule help us here? We need to start with some prior guess about the probability $P(S=s)$ : this is called our prior. We might assume the settings $S$ are equally likely to take any value in some range, or we might use the opinions of experts to make a more informed guess. Second, as mentioned, we can compute $P(M=m \mid S=s)$. Finally, the number $P(M=m)$ is independent of our choice of settings, so we can treat it as a constant. Thus, we can use Bayes' rule to compute $P(S=s \mid M=m)$ as a function of the settings $s$ - at least up to a constant factor. Then, since probabilities must sum to 1 , we can figure out this constant.

We call $P(S=s \mid M=m)$ the posterior, because it is the probability that the settings take some value 


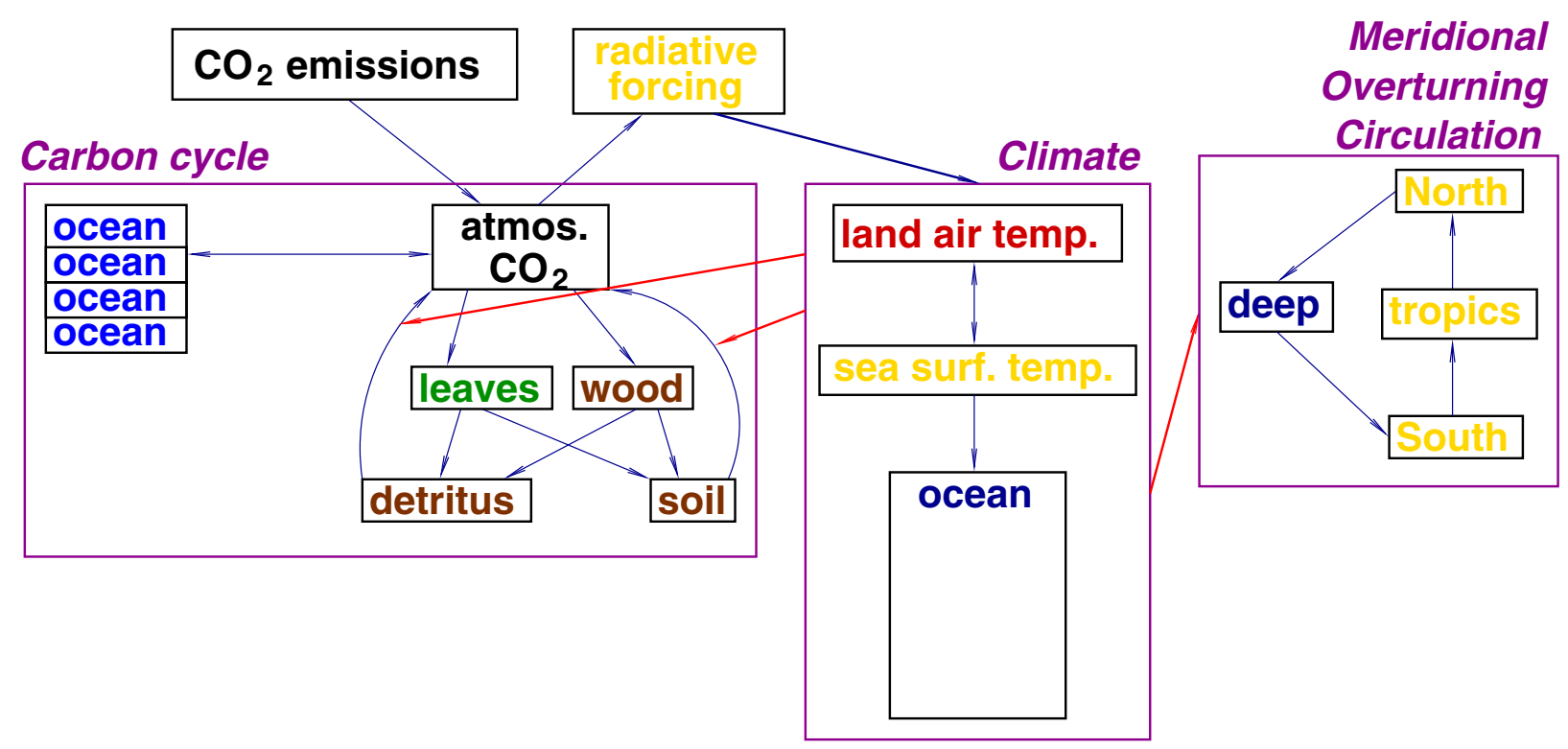

Figure 1: The box model used by Keller and Urban

after taking into account our measurements. Knowing this lets us do many things. It lets us find the most likely values of the parameters for our model, given our hard disc full of observed data. It also lets us find the probability that the settings lie within some set. This is important: if we're facing the possibility of a climate disaster, we don't just want to know the most likely outcome. We would like to know that with $95 \%$ probability, the outcome will lie in some range.

\section{An example}

Let us look at an example much simpler than that considered by Keller and Urban. Suppose our measurements are real numbers $m_{0}, \ldots, m_{T}$ related by

$$
m_{t+1}=s m_{t}-m_{t-1}+N_{t}
$$

Here $s$, a real constant, is our 'setting', while $N_{t}$ is some 'noise': an independent Gaussian random variable for each time $t$, with mean zero and some fixed standard deviation. Then the measurements $m_{t}$ will have roughly sinusoidal behavior but with irregularity added by the noise at each time step, as shown in Figure 2. Note how there is no clear signal from either the curves or the differences that the green curve is at the correct setting value while the blue one has the wrong one: the noise makes it nontrivial to estimate $s$. This is a baby version of the problem faced by Keller and Urban.

\section{Markov Chain Monte Carlo}

Having glibly said that we can compute the conditional probability $P(M=m \mid S=s)$, how do we actually do this? The simplest way would be to run our model many, many times with the settings set at $S=s$ and determine the fraction of times it predicts measurements consistent with $m$. This gives us an estimate of $P(M=m \mid S=s)$. Then we can use Bayes' rule to work out $P(S=s \mid M=m)$, at least up to a constant factor.

Doing this by hand would be incredibly time consuming and error prone, so computers are used for this task. In our example, we do this in Figure 3. As 


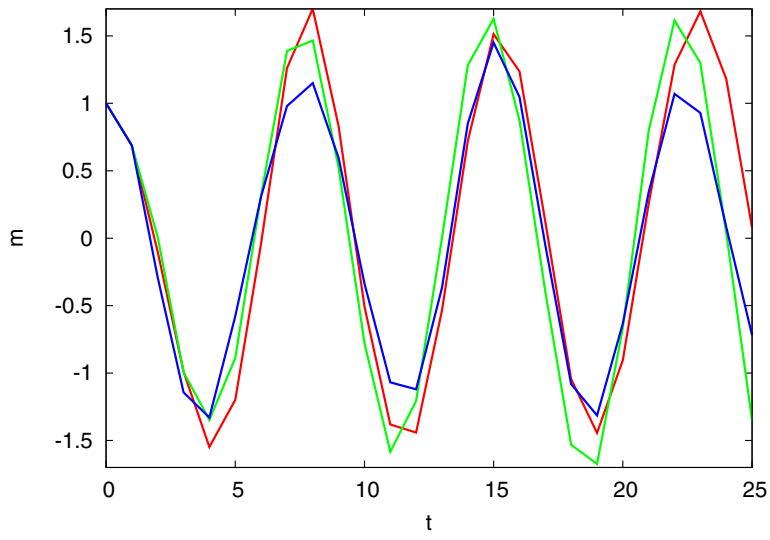

Figure 2: The example system: red are predicted measurements for a given value of the settings, green is another simulation for the same $s$ value and blue is a simulation for a slightly different $s$.

we keep running our model over and over, the curve showing $P(S=s \mid M=m)$ settles down to the right answer.

However, this is computationally inefficient, as shown in the probability distribution for small numbers of samples. This has quite a few 'kinks', which only disappear later. The problem is that there are lots of possible choices of $s$ to try. And this is for a very simple model!

When dealing with the 18 settings involved in the model of Keller and Urban, trying every combination would take far too long. A way to avoid this is Markov Chain Monte Carlo sampling. Monte Carlo is famous for its casinos, so a 'Monte Carlo' algorithm is one that uses random steps to converge upon the answer. A 'Markov chain' is a random walk: for example, where you repeatedly flip a coin and take one step right when you get a heads, and one step left when you get a tails. So, in Markov Chain Monte Carlo, we perform a random walk through the collection of all possible settings, collecting samples. From these samples we can estimate the density in the same way as before, only with greater computational efficiency.

The key to making this work is that at each step on the walk a proposed modification $s^{\prime}$ to the current

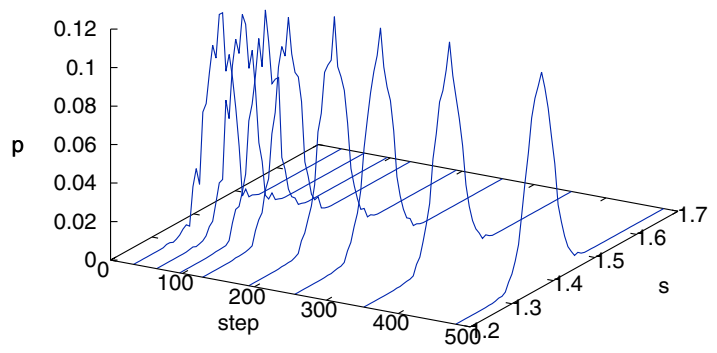

Figure 3: Estimates of the conditional probability $P(S=s \mid M=m)$, where at each step we run the model at all possible settings, and we keep a running total of the fraction of times each measurement $m$ occurs when the setting is $s$.

settings $s$ is generated randomly-but it may be rejected if it does not seem to improve the estimates. By cleverly choosing the rule for doing this, in a way motivated by Bayes' rule, the random walk can be designed so that in the long run it visits each setting $s$ with a frequency equal to to $P(S=s \mid M=m)$. With some additional tricks - such as discarding the very beginning of the walk - this gives a set of samples which can be used to estimate $P(S=s \mid M=m)$. For details, try the textbooks by Bolstad [3] or Press et al. [6].

Figure 4 shows the results of using the Markov Chain Monte Carlo procedure to figure out $P(S=$ $s \mid M=m)$ in our example. Compared to the method in Figure 3, we run the Markov Chain Monte Carlo method for more steps, but each step involves running the model at just one setting, instead of all possible settings, so it is really much more efficient.

The key advantage of Markov Chain Monte Carlo is that it avoids performing many simulations with settings where the probability is low, as we can see from the way the red path of the random walk stays under the big peak in the probability density almost all the time. More impressively, it achieves this without us needing to know ahead of time where this peak will 


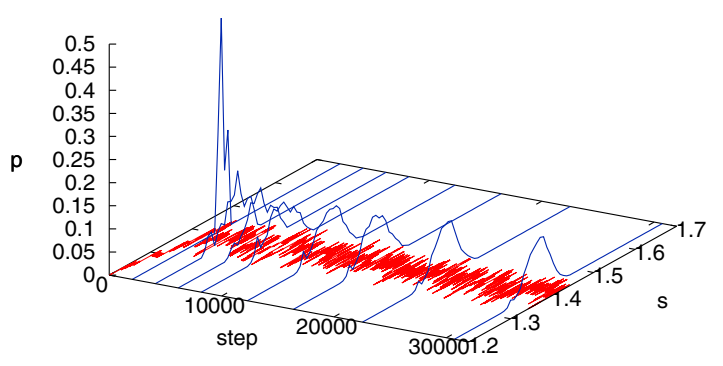

Figure 4: The estimates of $P(S=s \mid M=m)$ using Markov Chain Monte Carlo, showing the current distribution estimate at increasing intervals. The red line shows the current position of the random walk. Again the kinks are almost gone in the final distribution.

be! This becomes even more important as we move to dealing with more complicated models, where often the regions of high probability density are irregularly shaped multidimensional 'blobs', as it proves very effective at finding them efficiently.

Why is it worth doing so much work to estimate the probability distribution for settings for a climate model? One reason is that we can then estimate probabilities of future events, such as the collapse of the Atlantic Meridional Ocean Current. And what's the answer? According to Keller and Urban's calculation, this current will likely weaken by about a fifth in the 21st century, but a complete collapse is unlikely before 2300 . This claim needs to be checked in many ways - for example, using more detailed models. But the importance of the issue is clear, and we hope we have made the importance of good mathematical ideas for climate science clear as well.

\section{Exploring the topic}

The Azimuth Project is a group of scientists, engineers and computer programmers interested in ques- tions like this [2]. If you have questions, or want to help out, just email us. Versions of the computer programs we used in this paper available at [1].

Here are some practical issues to investigate:

- The collapse of the Atlantic Meridional Overturning Circulation would cause a temperature drop in Northern Europe. Research the effects on humans and ecosystems of drops of $1^{\circ}, 3^{\circ}$ and $7^{\circ}$ Celsius.

- Learn in detail how Markov Chain Monte Carlo works $[3,5,6]$. Also learn about some other methods of parameter estimation and try those in the example here.

- We've seen a one-dimensional system with one setting. Simulate some multi-dimensional and multi-setting systems. What new issues arise?

Acknowledgements. We thank Jan Galkowski, Graham Jones, Nathan Urban, and other members of the Azimuth Project for many helpful discussions.

\section{References}

[1] Software available at http://math.ucr.edu/home/baez/climate-math/.

[2] Azimuth Project, http://www.azimuthproject.org.

[3] W. M. Bolstad, Understanding Computational Bayesian Statistics, John Wiley, New York, 2010.

[4] K. Keller and N. Urban, Probabilistic hindcasts and projections of the coupled climate, carbon cycle and Atlantic meridional overturning circulation system: a Bayesian fusion of centuryscale measurements with a simple model, Tellus A 62 (2010), 737-750. Also available at http://public.lanl.gov/nurban/pubs/moc-projections.pdf.

[5] J. K. Kruschke, Doing Bayesian Data Analysis: A Tutorial with $R$ and BUGS, Academic Press, New York, 2010. 
[6] W. H. Press, S. A. Teukolsky, W. T. Vetterling and B. P. Flannery, Numerical Recipes: The Art of Scientific Computing, Section 15.8: Markov Chain Monte Carlo, Cambridge U. Press, Cambridge, 2007. Also available at http://apps.nrbook.com/empanel/index.html\#pg=824.

[7] Eliezer S. Yudkowsky, An intuitive explanation of Bayes' theorem, available at http://yudkowsky.net/rational/bayes. 